\title{
Closed-Form Bit Error Probabilities for FBMC
}

\section{Systems}

\author{
Ricardo Tadashi Kobayashi and Taufik Abrao \\ Department of Electrical Engineering (DEEL) State University of Londrina (UEL). \\ Po.Box 10.011, CEP:86057-970, Londrina, PR, Brazil. \\ Email: ricardokobayashi@uel.br; taufik@uel.br
}

\begin{abstract}
This paper analyses the data reconstruction effects emerged from the deployment of non-perfect prototype filters in Filter Bank MultiCarrier (FBMC) systems operating over Additive White Gaussian Noise (AWGN) and frequency-flat Rayleigh channels considering frequency-flatness for each subcarrier. This goal is attained by studying the Bit Error Rate (BER) effects of the prototype filters, increasing the scenario complexity progressively. Despite the complexity, both exact and approximate Bit Error Probability (BEP) expressions portray the BER degradation analytically for any FBMC prototype filter. Numerical results demonstrate that the proposed BEP expressions match perfectly with the simulated BER performance for FBMC systems, regardless of the prototype filter choice.
\end{abstract}

\section{Index Terms}

Bit error probabilities; Multicarrier systems; Prototype filters, FBMC design.

\section{INTRODUCTION}

With the ever-growing popularity of wireless communications, services have become increasingly complex and demanding in order to meet the stringent user requirements. Therefore, 5th Telecommunication Generation $(5 \mathrm{G})$ compliant technologies is a hot research topic at this point [1], [2]. Among them, we can name massive Multiple-Input Multiple-Output (MIMO) systems, mmWaves, and mMTC as the most promising technologies for 5G [3]. Massive MIMO systems

R. T. Kobayashi and T. Abrao are with the Department of Electrical Engineering (DEEL) State University of Londrina (UEL). Po.Box 10.011, CEP:86057-970, Londrina, PR, Brazil. Email: ricardokobayashi@uel.br; taufik@uel.br 
deploy a large number of antennas to enhance spectral/energy efficiency [4], while mmWaves devices operate in frequencies over $30 \mathrm{GHz}$ [5], where spectrum is abundant. Despite not being expected to be fully supported, basic concepts of Cognitive Radio can be useful for 5G [6] and other wireless systems to come, as spectrum usage can be considerably enhanced. Another aspect of utmost importance is the radio access, as spectral efficiency is primordial for dense networks.

Orthogonal Frequency-Division Multiplexing (OFDM) is a classical waveform, being deployed in contemporary standards such as 802.11 for local networks, 802.16 for Wimax and LTE-A for 4G systems [7]. Despite its popularity, such waveform presents some limitations. First, OFDM is known to present high Out-of-Band (OoB) emission due to its rectangular envelope. Moreover, OFDM channel equalization requires the usage of reasonably long Cyclic-Prefix (CP), reducing the spectral efficiency. Another drawback is the reliance of OFDM on its orthogonality, which prevents its efficient operation for random access channel. In order to address such issues, alternative multicarrier schemes have been intensively studied in the last years [8].

Among the waveforms alternatives, Filter Bank MultiCarrier (FBMC) is considered one of the most promising radio access for wireless systems to come [9]-[12]. One of the reasons for such prospect is the robustness of FBMC systems against InterSymbol Interference (ISI) without the usage of long CPs [13]. According to [14], FBMC systems present a synergistic operation with massive MIMO. Furthermore, FBMC systems are also known to provide very low OoB emission, favoring spectrum efficiency. Hence, such aspects made FBMC one of the waveforms selected for the METIS project [15] and the main choice for PHYDIAS project [16].

Although FBMC systems have been extensively studied in the last few years, they are still much less explored than OFDM, and there are still many open issues to be addressed. Despite their similarities, OFDM methods and algorithms are not fully compatible with FBMC systems as they rely on orthogonality in the real field. Hence, channel estimation is proceeded differently due to the imaginary interference emerged in FBMC systems [17], and Peak-to-Average Power Ratio (PAPR) methods such as the Tone Reservation also needs to be modified [18], [19].

The prototype filter choice is yet another subject to be addressed, as they control the overall spectrum and reconstruction features of FBMC systems. Despite the Mirabbasi-Martin filter [20], [21] being the most widespread prototype filter, there are plenty other options [22]-[30] that offer different OoB emission, time/frequency pulse dispersion, and Signal-to-Interference Ratio (SIR) trade-offs. 
As the deployment of different prototype filter yields different SIR levels, symbol reconstruction performance, i.e., Bit Error Rate (BER) performance is expected to be different. Despite the effects of the prototype filter on the overall performance of FBMC transmissions being extensively studied, the provision expressions for Bit Error Probability (BEP) have been ignored so far. Such subject remained ignored as prototype filters can be designed to achieve large SIR levels, meaning marginal effects on the BER performance. Moreover, one can also deploy SIR or distortion measurements to assess the effectiveness of the prototype filter on the overall transmission of an FBMC system qualitatively. However, beyond the aforementioned facts, the provision of closed-form BEPs has not been made yet due to their non-trivial solution. From this perspective, the main contribution of this paper lies in the provision of analytic expressions of BEP for FBMC systems, regardless of the prototype filter option. Hence, as an effective contribution and novelty, through the results of this work, we quantify the prototype filter effect on the BER performance of an FBMC system, which was known only qualitatively until now.

The rest of the paper is organized as follows. Section $\amalg$ presents the basics of FBMC systems. Section III discusses the foundations for evaluating BEP in both Additive White Gaussian Noise (AWGN) and frequency-flat Rayleigh channels. In Section IV, analytic formulas for evaluating the BEP of FBMC systems are proposed. Section $\nabla$ presents corroborative numerical results that compare the numerical BER with the derived BEP expressions. Finally, Section [VI] closes the paper by offering conclusions and final remarks. Notation: $\operatorname{Re}\{\cdot\}$ and $\operatorname{Im}\{\cdot\}$ are the real part and imaginary part operators, while $j=\sqrt{-1} .\lceil\cdot\rceil$ is the ceil operator. $\langle a[k] \mid b[k]\rangle=\sum_{k} a[k] b^{*}[k]$ is the inner product between $a[k]$ and $b[k]$, where $(\cdot)^{*}$ is the complex conjugation operator. $u(\cdot)$ and $\delta(\cdot)$ are, respectively, the step function and the Dirac delta function.

\section{FBMC MULTIPLEXING}

The FBMC multiplexing distributes phase-shifted Pulse Amplitude Modulation (PAM) symbols along $M$ subcarriers with a specific pulse shaping. The discrete representation of an FBMC signal consists of the superposition of PAM symbols shaped by their respective pulses, i.e.,

$$
s[k]=\sum_{n=-\infty}^{\infty} \sum_{m=0}^{M-1} a_{m, n} p_{m, n}[k],
$$


where $a_{m, n}$ is the $n$th PAM symbol of the $m$ th subcarrier and $p_{m, n}[k]$ is the associate pulse shape of length $L_{p} 1$, which is given by

$$
p_{m, n}[k]=p\left[k-n \frac{M}{2}\right] e^{j\left(\frac{2 \pi}{M} m \underline{k}+\phi_{m, n}\right)},
$$

where $p[k]$ is the prototype filter, $\underline{k}=k-\left(L_{p}-1\right) / 2$ and $\phi_{m, n}$ is the phase-shift added to $a_{m, n}$. Notice that $\phi_{m, n}$ is designed to make the adjacent symbols phase-shifted by $\pi / 2$ and typically set to

$$
\phi_{m, n}=\frac{\pi}{2}(m+n)
$$

which will be deployed throughout the remainder of this work.

\section{A. Symbol Reconstruction}

In order to recover the PAM symbols at the receiver side, the received FBMC signal must be correlated to the pulse of the desired symbol. Hence, for a noiseless scenario, the symbol $a_{m_{0}, n_{0}}$ can be retrieved by taking the real part of the $s[k]$ projection onto $p_{m_{0}, n_{0}}[k]$ :

$$
\tilde{a}_{m_{0}, n_{0}}=\operatorname{Re}\left\{\left\langle s[k] \mid p_{m_{0}, n_{0}}[k]\right\rangle\right\} .
$$

Notice that $\left\{p_{m, n}[k]\right\}$ is not strictly orthogonal, leading to symbol self-interference. By expanding eq. (4), one can evaluate the whole interference experienced by the $n_{0}$ th symbol of the $m_{0}$ th subcarrier:

$$
\tilde{a}_{m_{0}, n_{0}}=a_{m_{0}, n_{0}}+\sum_{\substack{n \neq n_{0} \\ m \neq m_{0}}} a_{m, n} \operatorname{Re}\left\{\left\langle p_{m, n}[k] \mid p_{m_{0}, n_{0}}[k]\right\rangle\right\} .
$$

Therefore, an appropriate prototype filter must be deployed in order to enable near-perfect reconstruction at the receiver, since it introduces the self-interference highlighted in eq. (5). Since the prototype filter choice can affect considerably symbol reconstruction, the characterization of this effect is very important and is investigated in this work through the provision of BEP expressions that describe such phenomenon.

Indeed, prototype filters can be designed to achieve perfect reconstruction, meaning no distortion during symbol reconstruction described in eq. (5). In this sense, a prototype filter is said to offer perfect reconstruction if it satisfies eq. (21) of [31]. Nevertheless, a small amount of distortion is almost always allowed to improve the spectral performance of prototype filters deployed in FBMC systems [21].

\footnotetext{
${ }^{1}$ Typical values for $L_{p}$ are $K M-1, K M$ and $K M+1$, where $K$ is the overlapping factor of the prototype filter.
} 


\section{B. Interference Elements}

As observed in eq. (5), the prototype filter introduces an interference on the symbol reconstruction procedure. Since this phenomenon degrades the BER performance, let us define

$$
\begin{aligned}
\epsilon_{m, n} & =\operatorname{Re}\left\{\left\langle p_{m, n}[k] \mid p_{0,0}[k]\right\rangle\right\}, \quad n, m \neq 0 \\
& =\cos \left(\phi_{m, n}\right) \sum_{k=-\infty}^{\infty} p\left[k-n \frac{M}{2}\right] p[k] \cos \left(\frac{2 \pi}{M} m \underline{k}\right)
\end{aligned}
$$

as the interference introduced by the symbol $a_{m, n}$ into the symbol $a_{0,0}$.

From (6), one can enumerate the following properties for the interference elements:

i. $\epsilon_{0,0}$ represents the pulse energy;

ii. $\epsilon_{m, n}$ is an even sequence concerning the index n, i.e., $\epsilon_{m, n}=\epsilon_{m,-n}$;

iii. $\epsilon_{m, n}$ is even circular symmetric concerning $m$, i.e., $\epsilon_{m, n}=-\epsilon_{M-m, n}$, for $1 \leq m \leq M / 2$;

iv. $\epsilon_{m, n}=0$ for $|n|>\left\lceil\frac{L_{p}}{M / 2}\right\rceil-1$, since the length of the prototype filter is finite;

v. $\epsilon_{m, n}=0$ case $m+n$ is odd, given the cosine term.

These five properties can be deployed to define

$$
\mathcal{E}=\left\{\begin{array}{l|l}
\epsilon_{m, n} & \begin{array}{l}
0 \leq m \leq M-1 \\
|n| \leq\left\lceil\frac{L_{p}-1}{M / 2}\right\rceil-1 \\
m+n \text { even } \\
m+n \neq 0
\end{array}
\end{array}\right\}
$$

as the set containing all the non zero interference elements $\epsilon_{m, n}$.

It is noteworthy mentioning that the interference set $\mathcal{E}$ contains

$$
|\mathcal{E}|=M\left(\left\lceil\frac{L_{p}-1}{M / 2}\right\rceil-\frac{1}{2}\right)-1
$$

elements and is a keypoint to derive the BEPs for FBMC systems deploying arbitrary prototype filters.

\section{III. $N_{p}$-PAM BEP: Single CARrier CASE}

In order to provide a comprehensive presentation of the subject, let us present first the single carrier case for calculating the BEP of the transmission of PAM symbols. Initially, $N_{p}$-PAM modulation is briefly reviewed. In the sequel, the BEPs are derived considering a single carrier transmission of $N_{p}$-PAM symbols over AWGN as well as frequency-flat Rayleigh channels. 


\section{A. PAM Modulation}

By deploying PAM, data symbols are constrained to the alphabet

$$
\mathcal{A}_{N_{p}}=\left\{-N_{p}+1,-N_{p}+3, \cdots, N_{p}-1\right\}
$$

meaning that only the in-phase component is deployed. Furthermore, one can easily infer that each $N_{p}$-PAM symbol can deliver

$$
N_{b}=\log _{2}\left(N_{p}\right)
$$

bits by using an average symbol energy of

$$
E_{s}=\frac{N_{p}^{2}-1}{3}
$$

\section{B. BEP for Single Carrier PAM Transmission over AWGN Channels}

First, consider the simplest scenario: the transmission of PAM symbols over an AWGN channel. Such a transmission can be modeled as

$$
x=a+\eta,
$$

where $a$ is the transmitted $N_{p}$-PAM symbol and $\eta$ is the additive noise described by the Probability Density Function (PDF)

$$
f_{\eta}(y)=\frac{1}{\sqrt{\pi N_{0}}} e^{-\frac{y^{2}}{N_{0}}}
$$

where $N_{0}$ is the noise power density. Furthermore, one can rewrite the noise power in terms of the normalized Signal-to-Noise Ratio (SNR) $\gamma_{t}^{2}$ based on eq. (10) and (11), i.e.,

$$
N_{0}=\frac{N_{p}^{2}-1}{3 \log _{2} N_{p} \gamma_{b}} .
$$

A classical and straightforward approximation for evaluating the BEP of PAM symbols corrupted by additive noise is given by [32]:

$$
P_{b}^{\mathrm{AWGN}, \mathrm{PAM}}\left(\gamma_{b}\right) \approx \frac{2\left(N_{p}-1\right)}{N_{p} \log _{2} N_{p}} \int_{1}^{\infty} f_{\eta}(z) d z .
$$

Despite being very simple, eq. (15) only considers symbol errors caused by adjacent symbols. Moreover, eq. (15) approximates the BEP as the Symbol Error Probability (SEP) divided by the number of bits. Thus, such expression considers that symbols are misestimated at the same rate of the bits.

${ }^{2}$ Bit energy per noise energy. 
By solving eq. (15) considering an AWGN channel, one can obtain the approximate BEP of an $N_{p}$-PAM transmission over an AWGN channel:

$$
P_{b}^{\mathrm{AWGN}, \mathrm{PAM}}\left(\gamma_{b}\right) \approx \frac{2\left(N_{p}-1\right)}{N_{p} \log _{2} N_{p}} Q\left(\sqrt{\frac{6 \log _{2} N_{p}}{N_{p}^{2}-1} \gamma_{b}}\right)
$$

where

$$
Q(y)=\frac{1}{\sqrt{2 \pi}} \int_{y}^{\infty} e^{-\frac{z^{2}}{2}} d z
$$

is the tail distribution of a standard normal distribution. Notice that an exact BEP expression is far more complex as all error combinations must be taken into account. Fortunately, authors of [33] offer the exact expression considering $N_{p}$-PAM Gray coded symbols:

$$
\begin{aligned}
P_{b}^{\mathrm{AWGN}, \mathrm{PAM}}\left(\gamma_{b}\right)= & \frac{1}{N_{p} \log _{2} N_{p}} \times \\
& \sum_{k=1}^{\log _{2} N_{p}} \sum_{i=0}^{\left(1-2^{-k}\right) N_{p}-1} w_{i, k, N_{p}} \int_{2 i+1}^{\infty} f_{\eta}(z) d z,
\end{aligned}
$$

where

$$
w_{i, k, N_{p}}=(-1)^{\left\lfloor i 2^{k-1} / N_{p}\right\rfloor}\left(2^{k-1}-\left\lfloor\frac{i 2^{k-1}}{N_{p}}+\frac{1}{2}\right\rfloor\right) .
$$

In particular, an AWGN scenario leads to the exact BEP

$$
\begin{aligned}
P_{b}^{\mathrm{AWGN}, \mathrm{PAM}}\left(\gamma_{b}\right) & =\frac{2}{N_{p} \log _{2} N_{p}} \sum_{k=1}^{\log _{2} N_{p}} \sum_{i=0}^{\left(1-2^{-k}\right) N_{p}-1} w_{i, k, N_{p}} \\
& \times Q\left((2 i+1) \sqrt{\frac{6 \log _{2} N_{p}}{N_{p}^{2}-1} \gamma_{b}}\right) .
\end{aligned}
$$

\section{BEP for Single Carrier PAM Transmission over Frequency-Flat Rayleigh Channels}

By considering that symbol transmission takes place over a Rayleigh channel, the transmission model presented in (12) must be rewritten as

$$
x=h a+\eta,
$$

where $h$ is the complex-valued channel coefficient.

Since the channel fades the signal randomly, one must consider all the possible values $h$ can take in order to evaluate the average BEP. Thus, the overall BEP is obtained by the integral

$$
P_{b}^{\mathrm{RAY}}\left(\gamma_{b}\right)=\int_{0}^{\infty} f_{|h|^{2}}\left(z, \gamma_{b}\right) P_{b}^{\mathrm{AWGN}}(z) d z
$$


where

$$
f_{|h|^{2}}\left(y, \gamma_{b}\right)=\frac{1}{\gamma_{b}} e^{-\frac{y}{\gamma_{b}}} u(y)
$$

is the PDF of the squared channel coefficient for a given normalized SNR $\gamma_{b}$.

By combining and solving eqs. (22) and (16), it can be concluded that an approximation for the BEP of $N_{p}$-PAM symbols transmitted over frequency-flat Rayleigh channels is given by

$$
P_{b}^{\mathrm{RAY}, \mathrm{PAM}}\left(\gamma_{b}\right) \approx \frac{N_{p}-1}{N_{p} \log _{2} N_{p}}\left(1-\frac{\sqrt{\frac{3 \log _{2} N_{p}}{N_{p}^{2}-1} \gamma_{b}}}{\sqrt{\frac{3 \log _{2} N_{p}}{N_{p}^{2}-1} \gamma_{b}+1}}\right) .
$$

Similarly, an exact formula can be obtained through eqs. (22) and (20):

$$
\begin{aligned}
P_{b}^{\mathrm{RAY}, \mathrm{PAM}}\left(\gamma_{b}\right)= & \frac{1}{N_{p} \log _{2} N_{p}} \sum_{k=1}^{\log _{2} N_{p}} \sum_{i=0}^{\left(1-2^{-k}\right) N_{p}-1} w_{i, k, N_{p}} \\
& \times\left(1-\frac{\sqrt{\frac{3(2 i+1)^{2} \log _{2} N_{p}}{N_{p}^{2}-1} \gamma_{b}}}{\sqrt{\frac{3(2 i+1)^{2} \log _{2} N_{p}}{N_{p}^{2}-1} \gamma_{b}+1}}\right),
\end{aligned}
$$

which was proposed by [34].

At this point, it is noteworthy mentioning that the expressions presented in this section can be modified to characterize OFDM which is the baseline multiplexing scheme deployed in most multicarrier comparisons. First, notice that OFDM deploys $N_{q}$-Quadrature Amplitude Modulation (QAM) symbols, while the BEP expressions presented previously are valid for $M_{p}$-PAM. This issue can be solved by using $N_{p}=\sqrt{N_{q}}$ in the previous equations, as QAM can be interpreted as the independent transmission of PAM symbols over the in-phase and quadrature components. Moreover, OFDM systems deploy CP, which reduces the overall SNR of the signal and increases the error rate. In this sense, the OFDM BEP can be expressed by

$$
\begin{aligned}
P_{b}^{\mathrm{AWGN,OFDM}}\left(\gamma_{b}\right)= & \frac{2}{\sqrt{N_{q}} \log _{2} \sqrt{N_{q}}} \sum_{k=1}^{\log _{2} \sqrt{N_{q}}} \sum_{i=0}^{\left(1-2^{-k}\right) \sqrt{N_{q}}-1} w_{i, k, \sqrt{N_{q}}} \\
& \times Q\left((2 i+1) \sqrt{\frac{3 \log _{2} N_{q}}{N_{q}-1} \frac{M}{M+N_{c p}} \gamma_{b}}\right.
\end{aligned}
$$

for AWGN and by

$$
\begin{aligned}
P_{b}^{\mathrm{RAY}, \text { OFDM }}\left(\gamma_{b}\right)= & \frac{1}{\sqrt{N_{q}} \log _{2} \sqrt{N_{q}}} \sum_{k=1}^{\log _{2} \sqrt{N_{q}}} \sum_{i=0}^{\left(1-2^{-k}\right) \sqrt{N_{q}}-1} w_{i, k, \sqrt{N_{q}}} \\
& \times\left(1-\frac{\sqrt{\frac{3(2 i+1)^{2} \log _{2} N_{q}}{2\left(N_{q}-1\right)} \frac{M}{M+N_{c p}} \gamma_{b}}}{\sqrt{\frac{3(2 i+1)^{2} \log _{2} N_{q}}{2\left(N_{q}-1\right)} \frac{M}{M+N_{c p}} \gamma_{b}+1}}\right)
\end{aligned}
$$

for flat-frequency Rayleigh channels 


\section{IV. $N_{p}$-PAM BEP: FBMC CASE}

Based on the foundation provided previously, this section builds up the closed-form BEP expressions for FBMC systems over AWGN and frequency-flat Rayleigh channels.

\section{A. BEP for FBMC Transmission over AWGN Channels}

For the FBMC case over AWGN, one must combine the multiplexed signal with the effect of the additive noise, i.e.,

$$
x[k]=s[k]+\eta[k],
$$

where $\eta[k] \sim \mathcal{C N}\left(0, N_{0}\right)$. Hence, the reconstruction of the $n_{0}$ th symbol of the $m_{0}$ th subcarrier can be proceeded by combining eqs. (28) and (4), i.e.,

$$
\begin{aligned}
x_{m_{0}, n_{0}}= & \operatorname{Re}\left\{\left\langle x[k] \mid p_{m_{0}, n_{0}}[k]\right\rangle\right\} \\
& a_{m_{0}, n_{0}} \\
& +\sum_{\substack{n \neq n_{0} \\
m \neq m_{0}}} a_{m, n} \epsilon_{m, n}+\operatorname{Re}\left\{\left\langle\eta[k] \mid p_{m_{0}, n_{0}}[k]\right\rangle\right\} \\
= & a_{m_{0}, n_{0}}+\epsilon_{0}+\eta_{0},
\end{aligned}
$$

where $\epsilon_{0}$ is the overall interference over $a_{m_{0}, n_{0}}$. At this point, one can observe that the easiest option to derive the BEP of an FBMC signal under AWGN signal is achieved by

i. obtaining the PDF of the noise combined with the interference $\left(\epsilon_{0}+\eta_{0}\right)$;

ii. deploying eq. (15) for an approximate BER expression or (20) for the exact BER formula derivation.

1) PDF of the Noise plus Interference : By recalling that the resulting PDF of the sum of two random variables is the convolution of both PDFs, i.e.,

$$
f_{a+b}(y)=\int_{-\infty}^{\infty} f_{a}(z) f_{b}(y-z) d z .
$$

Thus, since the PDF of the $n$th PAM symbol of the $m$ th subcarrier is modeled by

$$
f_{a_{m, n}}(y)=\frac{1}{N_{p}} \sum_{a_{m, n} \in \mathcal{A}_{N_{p}}} \delta\left(y-a_{m, n}\right)
$$

and $\epsilon_{m, n}$ is deterministic, one may conclude that

$$
f_{a_{m, n} \epsilon_{m, n}}(y)=\frac{1}{N_{p}} \sum_{a_{m, n} \in \mathcal{A}_{N_{p}}} \delta\left(y-a_{m, n} \epsilon_{m, n}\right) .
$$


Notice that the summation notation adopted in eqs. (31) and 32 provides a more compact presentation. In this notation, the summation includes all the elements of the $N_{p}$-PAM set described in eq. (9).

According to eq. (29), the interference experienced at the receiver side $\left(\epsilon_{0}\right)$ is the combination of adjacent symbols $a_{m, n}$ scaled by their respective interference elements $\epsilon_{m, n}$. Hence, a series of convolutions must be evaluated in order to evaluate the PDF of $\epsilon_{0}$, which leads to

$$
\begin{aligned}
f_{\epsilon_{0}}(y)= & \frac{1}{N_{p}^{|\mathcal{E}|}} \sum_{a_{0,-2 K+1} \in \mathcal{A}_{N_{p}}} \ldots \sum_{a_{M-1,2 K-1} \in \mathcal{A}_{N_{p}}} \\
& \delta\left(y-\sum_{(m, n) \in \mathcal{E}} a_{m, n} \epsilon_{m, n}\right),
\end{aligned}
$$

where the last summation includes all the elements of $\mathcal{E}$, which are defined in eq. (7). Once more, such a notation was adopted in favor of a more compact presentation. Notice that eq. (33) has a large number of coefficients as it takes into account all possible combination of interfering elements $a_{m, n} \epsilon_{m, n}$. In this sense, we limited the last summation to the set $\mathcal{E}$ in order to ignore null interfering elements and decrease the complexity of the equation.

It is noteworthy mentioning that the increasing number of elements on the PDF of the summation of random variables can be observed in other random processes. For example, this behavior can also be observed on the Bates and Irwin-Hall distributions [35], [36], which consists of the summation of an arbitrary number of uniform random variables.

Since $f_{\epsilon_{0}}(x)$ is composed exclusively by Dirac delta functions, convolving it with any other function is trivial as the Dirac Function is the neutral element for convolution operation. Thus, the overall PDF of the noise plus interference can be expressed by (34). Therefore, by combining eq. (34) with eq. (15), the approximate BEP for FBMC signals over AWGN is obtained as (35). On the other hand, the exact BEP expression is obtained by combining eqs. (34) and (20), resulting in eq. (36).

\section{B. BEP for FBMC Transmission over Frequency-Flat Rayleigh Channels}

The received signal of an FBMC system operating over frequency-flat Rayleigh channels can be expressed as

$$
x[k]=\sum_{\ell=0}^{\infty} h[\ell] s[k-\ell]+\eta[k],
$$




$$
\begin{aligned}
& f_{\epsilon_{0}+\eta_{0}}(y)=\frac{1}{N_{p}^{\mid \mathcal{E}}} \sqrt{\frac{1}{2 \pi} \frac{6 \log _{2} N_{p}}{N_{p}^{2}-1} \gamma_{b}} \sum_{a_{0,-2 K+1} \in \mathcal{A}_{N_{p}}} \ldots \sum_{a_{M-1,2 K-1} \in \mathcal{A}_{N_{p}}} \exp \left[-\frac{6 \log _{2} N_{p}}{N_{p}^{2}-1} \gamma_{b}\left(\frac{y-\sum_{(m, n) \in \mathcal{E}} a_{m, n} \epsilon_{m, n}}{2}\right)^{2}\right] \\
& P_{b}^{\mathrm{AWGN}, \mathrm{FBMC}}\left(\gamma_{b}\right) \approx \frac{2\left(N_{p}-1\right)}{N_{p}^{|\mathcal{E}|+1} \log _{2} N_{p}} \sum_{a_{0,-2 K+1} \in \mathcal{A}_{N_{p}}} \cdots \sum_{a_{M-1,2 K-1} \in \mathcal{A}_{N_{p}}} Q\left[\sqrt{\frac{6 \log _{2} N_{p}}{N_{p}^{2}-1} \gamma_{b}}\left(1-\sum_{(m, n) \in \mathcal{E}} a_{m, n} \epsilon_{m, n}\right)\right]
\end{aligned}
$$

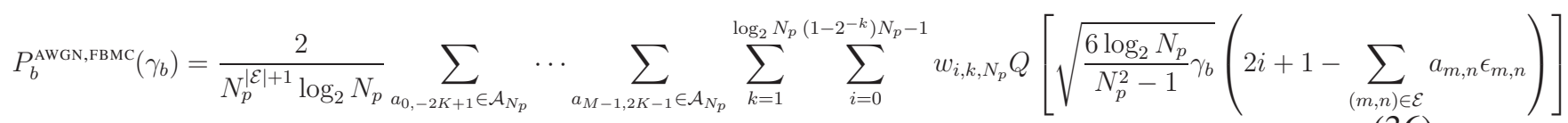

$$
\begin{aligned}
& \text { (36) } \\
& P_{b}^{\mathrm{RAY}, \mathrm{FBMC}}\left(\gamma_{b}\right) \approx \frac{N_{p}-1}{N_{p}^{|\mathcal{E}|+1} \log _{2} N_{p}} \sum_{a_{0,-2 K+1} \in \mathcal{A}_{N_{p}}} \cdots \sum_{a_{M-1,2 K-1} \in \mathcal{A}_{N_{p}}}\left[1-\left(2 i+1-\sum_{(m, n) \in \mathcal{E}} a_{m, n} \epsilon_{m, n}\right)\left[\frac{\frac{6 \log _{2} N_{p}}{N_{p}^{2}-1} \gamma_{b}}{\left(2 i+1-\sum_{(m, n) \in \mathcal{E}}\right.}\right]\right. \\
& P_{b}^{\mathrm{RAY}, \mathrm{FBMC}}\left(\gamma_{b}\right)=\frac{1}{N_{p}^{|\mathcal{E}|+1} \log _{2} N_{p}} \sum_{a_{0,-2 K+1} \in \mathcal{A}_{N_{p}}} \cdots \sum_{a_{M-1,2 K-1} \in \mathcal{A}_{N_{p}}} \sum_{k=1}^{\log _{2} N_{p}\left(1-2^{-k}\right) N_{p}-1} \sum_{i=0} w_{i, k, N_{p}} \\
& \times\left[1-\left(2 i+1-\sum_{(m, n) \in \mathcal{E}} a_{m, n} \epsilon_{m, n}\right) \sqrt{\left.\frac{\frac{6 \log _{2} N_{p}}{N_{p}^{2}-1} \gamma_{b}}{\left(2 i+1-\sum_{(m, n) \in \mathcal{E}} a_{m, n} \epsilon_{m, n}\right)^{2} \frac{6 \log _{2} N_{p}}{N_{p}^{2}-1} \gamma_{b}+1}\right]}\right]
\end{aligned}
$$

where $h[k]$ is the channel impulse response, which fades the transmitted signal and may introduce frequency selectivity. However, let us consider the simplifying assumption that each subchannel is frequency-flat. Such an assumption is plausible as multicarrier systems are typically designed to enable a simple one-tap per-subchannel equalization. Notice, however, that multi-tap equalizers [37]-[39] can be deployed as an alternative in scenarios where the number of subcarriers is insufficient to lead to subchannel frequency-flatness.

Aiming to retrieve the conveyed data, the received signal is pre-processed using the appropriate 
FBMC pulse, leading to

$$
\begin{aligned}
x_{m_{0}, n_{0}}= & \operatorname{Re}\left\{\left\langle x[k] \mid p_{m_{0}, n_{0}}[k]\right\rangle\right\} \\
= & H_{m_{0}}\left(a_{m_{0}, n_{0}}+\sum_{\substack{n \neq n_{0} \\
m \neq m_{0}}} a_{m, n} \epsilon_{m, n}\right) \\
& +\operatorname{Re}\left\{\left\langle\eta[k] \mid p_{m_{0}, n_{0}}[k]\right\rangle\right\} \\
\approx & H_{m_{0}}\left(a_{m_{0}, n_{0}}+\epsilon_{0}\right)+\eta_{0},
\end{aligned}
$$

where $H_{m_{0}}$ is the frequency response of the $m_{0}$ th subcarrier. Notice that eq. (40) is an approximation, as one can recall that the subchannels were considered frequency-flat. Moreover, eq. (40) is composed of the faded symbol plus the interference and the additive noise. From this perspective, the approximate BEP expression for an FBMC system operating over frequency-flat Rayleigh channels can be obtained by integrating eq. (22) considering eq. (35), which leads to eq. (37), while the exact expression emerges from the integration of eq. (22) considering eq. (36), yielding (38).

\section{Numerical Results}

This section presents the numerical results aiming to demonstrate the effectiveness of the BEP expressions derived throughout this paper. In order to achieve such a goal, we compare the derived BEP expressions with the simulated BER for the OFDM and FBMC setups described in Table \. considering both AWGN and frequency-flat Rayleigh channels. For FBMC, two prototype filters were deployed, the Extended Gaussian Function (EGF) and the Mirabbasi-Martin filter. Notice that the EGF [40] was chosen as the prototype filter due to its flexibility, which enables achieving different self-interference levels by tuning the spreading factor $\alpha$. This feature is useful to test the effectiveness of the derived expressions under very distinct scenarios. In particular, the EGF set with $\alpha=1.00$ provides a large SIR of $60.49 \mathrm{~dB}$, enabling near-perfect symbol reconstruction. On the other hand, by setting $\alpha=0.25$, the SIR of the EGF is reduced to $21.27 \mathrm{~dB}$, leading to a poor BER performance. Moreover, the Mirabbasi-Martin prototype filter is also deployed in this comparison as it presents a high spectrum and symbol reconstruction performances. Indeed, such a prototype filter is one of the most popular choices, being recommended in the PHYDIAS project [16]. 
Table I

PARAMETERS FOR THE FBMC SYSTEM.

\begin{tabular}{rll}
\hline Parameter & OFDM & FBMC \\
\hline Modulation order & 64 -QAM & 8 -PAM \\
Subcarriers, $M$ & 16 & 16 \\
Cyclic Prefix & $M / 8$ & 0 \\
Overlapping factor, $K$ & - & 4 \\
Filter length, $L_{p}$ & - & $M K+1$ \\
Prototype Filter & - & EGF, $\alpha=\{0.25,1.00\}$, \\
& & Mirabbasi-Martin \\
\hline
\end{tabular}

\section{A. Complexity Considerations}

Before presenting the BEP results, let us discuss the complexity of the derived expressions. Despite not relying on complex functions, the main issue of the derived expressions is the large number of terms of the summations. A more careful analysis reveals that the BEP expressions in eq. (35), (36), (37) and (38) presents at least $N_{p}^{|\mathcal{E}|+1}$ terms. Hence, the summation of a huge number of terms is required in order to provide the theoretical BEP expression. As an example, evaluating the BEP of the reasonably small system portrayed in Table \ would require the evaluation of approximately $3 \cdot 10^{107}$ terms. However, the interference elements $\epsilon_{m, n}$ decay rapidly as shown in Fig. 1, Therefore, let us restrain the set $\mathcal{E}$ to the 8 largest elements in order to make the developed expressions feasible and therefore useful. Notice that even with the limited set, the theoretical expressions require the summation of, at least, $16 \cdot 10^{6}$ elements.

\section{B. BER Performance}

In Figures 2.(a) and 2.(b), we present both the simulated BER and the theoretical BEP for the FBMC system described in Table \ considering an EGF prototype filter, and AWGN and frequency-flat Rayleigh scenarios. With $\alpha=1.00$ the FBMC system showed no apparent performance loss, whereas with $\alpha=0.25$, the BER performance is considerably severed, leading to a BER floor around $10^{-3}$ in the Rayleigh scenario. Such behavior is established due to the degradation of the prototype filter SIR, which dictates the amount of self-interference generated by the prototype filter. However, it is important to state that the prototype filter SIR improves as $\alpha$ increases, while the spectrum gets increasingly poor. As an example, EGFs with $\alpha$ values 


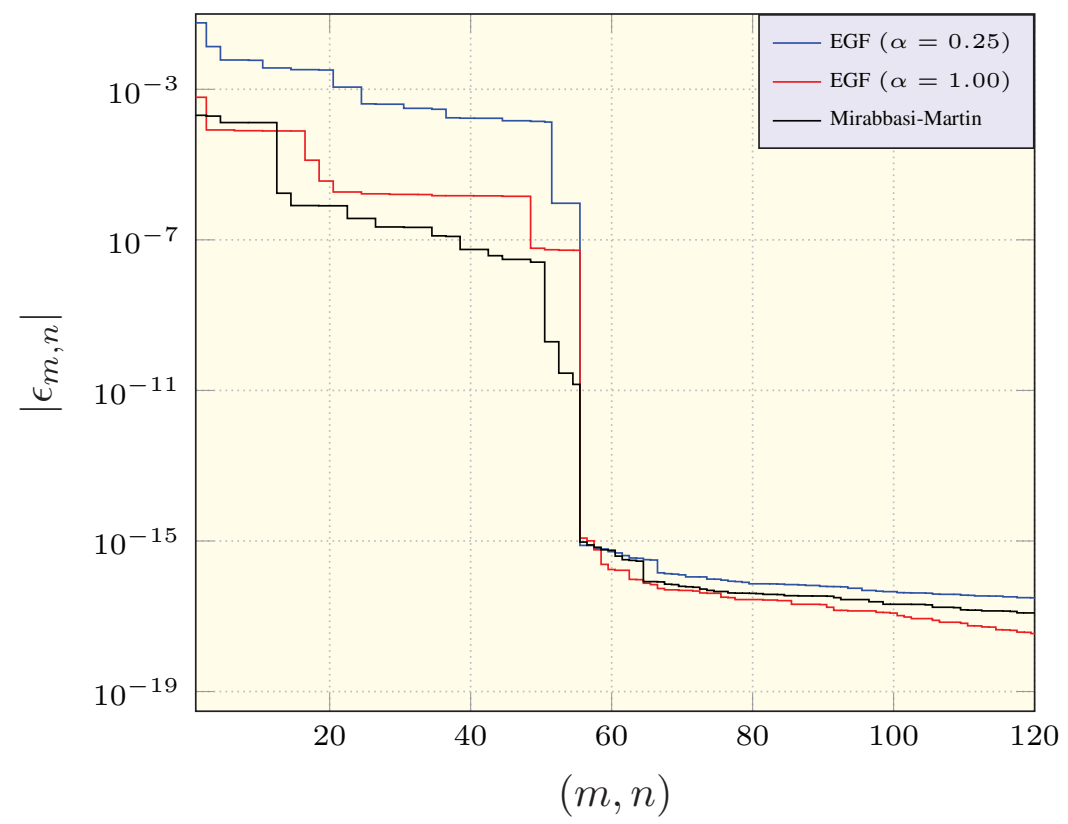

Figure 1. Ordered absolute values for the interference elements $\epsilon_{m, n}$ for the FBMC system described in Table प

of $0.5,1$ and 2 lead to SIR levels of 33.73, 60.49 and $114.48 \mathrm{~dB}$, respectively, whereas the OoB energy emission increases gradually to $-33.95,-19.69$ and $-12.46 \mathrm{~dB}$ [30].

In order to prove the effectiveness of the derived expression for any prototype filter, Figures 3. (a) and 3. (b) compare the BER with the BEP for an FBMC system deploying a MirabbasiMartin prototype filter [21] and the parameters described in Table II. Once more, the simulation and the analytically results depicted in Figures 3. (a) and 3.(b) are very close, proving the effectiveness of the proposed BEP expressions. Moreover, since the Mirabbasi-Martin prototype filter presents a high SIR level, i.e., $\approx 65.25 \mathrm{~dB}$, performance losses were not observed in Figures 3. (a) and 3.,(b).

Regarding the effectiveness of the derived formulas, one can observe that such expressions are very close to the simulation results. Despite reducing the size of the set $\mathcal{E}$ considerably, such approximation led a small influence on the overall results, as $\epsilon_{m, n}$ elements decay rapidly. Thus, the provided expression demonstrated to be effective to predict the BER performance of FBMC systems operating over AWGN and Rayleigh channels, regardless of the prototype filter choice.

Additionally, one can observe that the numerical results for OFDM match with the values found by the BEP expressions provided in section III. Compared to FBMC with EGF with $\alpha=1$ and Mirabbasi-Martin prototype filters, the BER performance of OFDM presents a small 


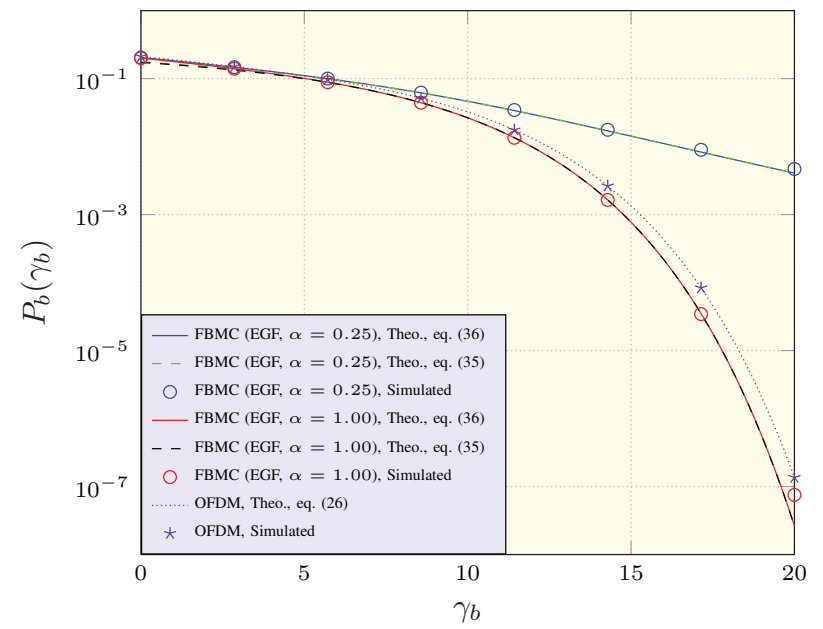

(a) AWGN

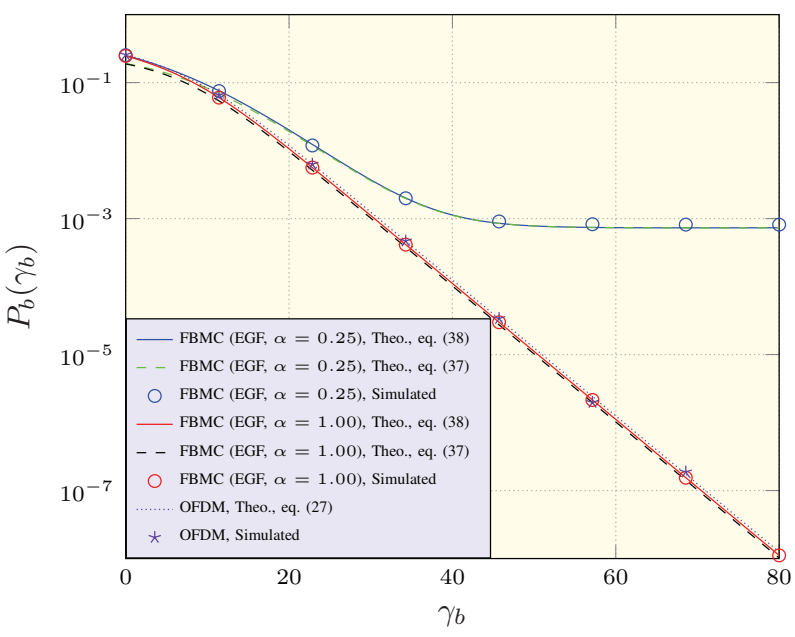

(b) Rayleigh

Figure 2. Theoretical and simulated BER for the FBMC system described in Table $\$ and with EGF prototype filter.

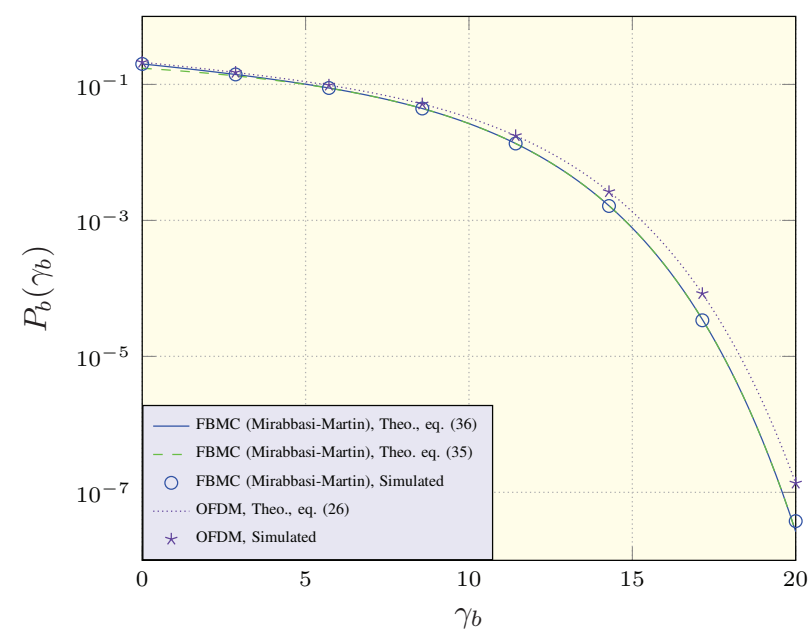

(a) AWGN

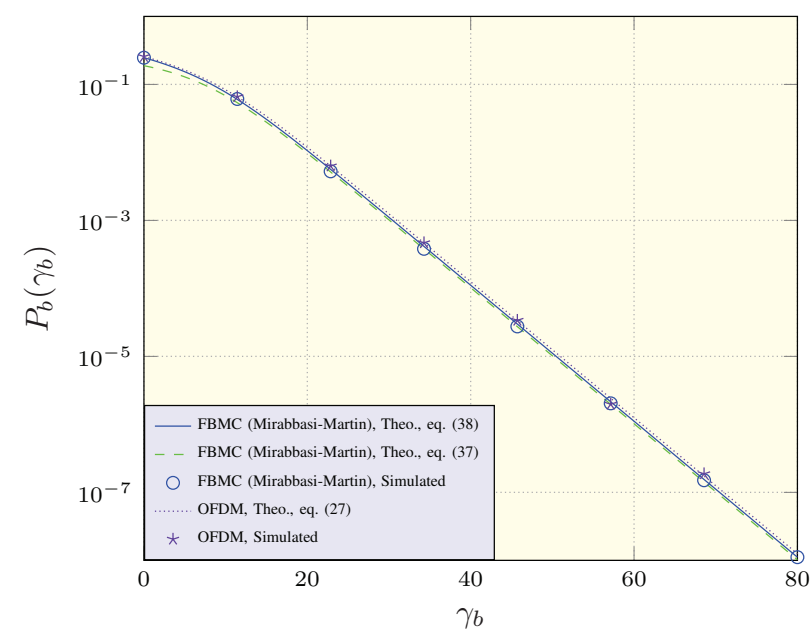

(b) Rayleigh

Figure 3. Theoretical and simulated BER for the FBMC system described in Table 1 and with Mirabbasi-Martin prototype filter.

offset due to the CP deployment, which does not carry actual data, reducing the overall SNR of the signal. On the other hand, prototype filters with low symbol reconstruction performance, e.g., EGF with $\alpha=0.25$, can limit the BER performance of FBMC systems operating under high SNR regime as observed in Figure 2.(b). 


\section{CONCLUSIONS}

Throughout this work, we derived the theoretical BEP expressions for FBMC systems under AWGN and frequency-flat Rayleigh channels, regardless of the prototype filter choice. Despite the required computational burden processing, the provided formulas are exact. Fortunately, the derived expressions have resulted relatively accurate yet by restricting the interference set $\mathcal{E}$ to the most significant values. Thus, such an approximation does not compromise the final BEP prediction values. From this perspective, the numerical results demonstrate that the proposed BEP expressions can adequately characterize the BER performance of FBMC systems. Thus, this work quantifies the effect of the prototype filter on the overall BER performance of FBMC systems, which until now was only known qualitatively in terms of dependencies on SIR and distortion measurements.

\section{ACKNOWLEDGMENTS}

This study was financed in part by the National Council for Scientific and Technological Development (CNPq) of Brazil under grants 404079/2016-4 and 304066/2015-0, in part by the CAPES - Brazil - Finance Code 001 (PhD scholarship), and in part by Londrina State University, Parana State Government. 


\section{LIST OF ACRONYMS}

BER Bit Error Probability

CP Cyclic Prefix

DFE Decision Feedback Equalizer

DPSS Discrete Prolate Spheroidal Sequences

DTFT Discrete-Time Fourier Transform

EGF Extended Gaussian Function

FBMC Filter Bank Multi-Carrier

GFDM Generalized Filtered-Division Filtered Multi-Carrier

IB In-Band

ICI Inter-Carrier Interference

ISI Inter-Symbol Interference

MMSE Minimum Mean-Square-Error

MSA Minimum Stopband Attenuation

OFDM Orthogonal Frequency-Division Multiplexing

OFDP Optimal Finite Duration Pulses

OOB Out-of-Band

PAM Pulse Amplitude Modulation

PAPR Peak-to-Avarage Power Ratio

QAM Quadrature Amplitude Modulation

QCQP Quadratically Constrained Quadratic Program

QP Quadratic Program

RMS Root Mean Square

SDP SemiDefinite Program

SIR Signal-to-Interference Ratio

SNR Signal-to-Noise Interference

SOCP Second Order Conic Program

SRRC Square-Root Raised-Cosine

UFMC Universal Filtered Multi-Carrier

V-BLAST Vertical Bell Laboratories Layered Space-Time 


\section{REFERENCES}

[1] M. Shafi, A. F. Molisch, P. J. Smith, T. Haustein, P. Zhu, P. D. Silva, F. Tufvesson, A. Benjebbour, and G. Wunder, "5G: a Tutorial Overview of Standards, Trials, Challenges, Deployment, and Practice," IEEE Journal on Selected Areas in Communications, vol. 35, no. 6, pp. 1201-1221, June 2017.

[2] J. Wang, A. Jin, D. Shi, L. Wang, H. Shen, D. Wu, L. Hu, L. Gu, L. Lu, Y. Chen, J. Wang, Y. Saito, A. Benjebbour, and Y. Kishiyama, "Spectral Efficiency Improvement with 5G Technologies: Results from Field Tests," IEEE Journal on Selected Areas in Communications, vol. 35, no. 8, pp. 1867-1875, August 2017.

[3] F. Boccardi, R. W. Heath, A. Lozano, T. L. Marzetta, and P. Popovski, "Five Disruptive Technology Directions for 5G," IEEE Communications Magazine, vol. 52, no. 2, pp. 74-80, February 2014.

[4] Q. Nadeem, A. Kammoun, M. Debbah, and M. Alouini, "Design of 5G Full Dimension Massive MIMO Systems," IEEE Transactions on Communications, vol. 66, no. 2, pp. 726-740, February 2018.

[5] T. S. Rappaport, Y. Xing, G. R. MacCartney, A. F. Molisch, E. Mellios, and J. Zhang, "Overview of Millimeter Wave Communications for Fifth-Generation (5G) Wireless Networks?with a Focus on Propagation Models," IEEE Transactions on Antennas and Propagation, vol. 65, no. 12, pp. 6213-6230, December 2017.

[6] S. Sasipriya and R. Vigneshram, "An Overview of Cognitive Radio in 5G Wireless Communications," in 2016 IEEE International Conference on Computational Intelligence and Computing Research (ICCIC), December 2016, pp. 1-5.

[7] T. Hwang, C. Yang, G. Wu, S. Li, and G. Y. Li, “OFDM and Its Wireless Applications: a Survey,” IEEE Transactions on Vehicular Technology, vol. 58, no. 4, pp. 1673-1694, May 2009.

[8] G. Wunder, P. Jung, M. Kasparick, T. Wild, F. Schaich, Y. Chen, S. T. Brink, I. Gaspar, N. Michailow, A. Festag, L. Mendes, N. Cassiau, D. Ktenas, M. Dryjanski, S. Pietrzyk, B. Eged, P. Vago, and F. Wiedmann, "5GNOW: NonOrthogonal, Asynchronous Waveforms for Future Mobile Applications,” IEEE Communications Magazine, vol. 52, no. 2, pp. 97-105, February 2014.

[9] B. F. Boroujeny, “OFDM Versus Filter Bank Multicarrier," IEEE Signal Processing Magazine, vol. 28, no. 3, pp. 92-112, May 2011.

[10] A. I. Perez-Neira, M. Caus, R. Zakaria, D. Le Ruyet, E. Kofidis, M. Haardt, X. Mestre, and Y. Cheng, "MIMO Signal Processing in Offset-QAM Based Filter Bank Multicarrier Systems," IEEE Transactions on Signal Processing, vol. 64, no. 21, pp. 5733-5762, Nov 2016.

[11] Y. Cai, Z. Qin, F. Cui, G. Y. Li, and J. A. McCann, "Modulation and Multiple Access for 5G Networks," IEEE Communications Surveys Tutorials, vol. 20, no. 1, pp. 629-646, Firstquarter 2018.

[12] B. F. Boroujeny, "Filter Bank Multicarrier Modulation: a Waveform Candidate for 5G and Beyond," Advances in Electrical Engineering, vol. 28, no. 3, pp. 92-112, May 2014.

[13] L. Zhang, P. Xiao, A. Zafar, A. u. Quddus, and R. Tafazolli, "FBMC System: An Insight into Doubly Dispersive Channel Impact," IEEE Transactions on Vehicular Technology, vol. 66, no. 5, pp. 3942-3956, May 2017.

[14] A. Farhang, N. Marchetti, F. Figueiredo, and J. P. Miranda, "Massive MIMO and Waveform Design for 5th Generation Wireless Communication Systems," in 1st International Conference on $5 G$ for Ubiquitous Connectivity. Akaslompolo, Finland: IEEE, November 2014, pp. 70-75.

[15] M. Schellmann, "Mobile and Wireless Communications Enablers for the Twenty-Twenty Information Society (METIS)," Tech. Rep. ICT-317669-METIS/D2.4 Proposed, February 2015.

[16] A. Viholainen, M. Bellanger, and M. Huchard, "Phydyas 007 - Physical Layer for Dynamic Access and Cognitive Radio," Tech. Rep. ICT-211887, January 2009. 
[17] W. Cui, D. Qu, T. Jiang, and B. Farhang-Boroujeny, "Coded Auxiliary Pilots for Channel Estimation in FBMC-OQAM Systems," IEEE Transactions on Vehicular Technology, vol. 65, no. 5, pp. 2936-2946, May 2016.

[18] Y. Rahmatallah and S. Mohan, "Peak-to-Average Power Ratio Reduction in OFDM Systems: a Survey and Taxonomy," Communications Surveys \& Tutorials, IEEE, vol. 15, no. 4, pp. 1567-1592, 2013.

[19] S. S. K. C. Bulusu, H. Shaiek, and D. Roviras, "Reduction of PAPR of FBMC-OQAM Systems by Dispersive Tone Reservation Technique," in 2015 International Symposium on Wireless Communication Systems (ISWCS). Brussels, Belgium: IEEE, August 2015, pp. 561-565.

[20] K. W. Martin, "Small Side-Lobe Filter Design for Multitone Data-Communication Applications," IEEE Transactions on Circuits and Systems II: Analog and Digital Signal Processing, vol. 45, no. 8, pp. 1155-1161, August 1998.

[21] S. Mirabbasi and K. Martin, "Overlapped Complex-Modulated Transmultiplexer Filters with Simplified Design and Superior Stopbands," IEEE Transactions on Circuits and Systems II: Analog and Digital Signal Processing, vol. 50, no. 8, pp. 456469, August 2003.

[22] A. Sahin, I. Guvenc, and H. Arslan, “A Survey on Multicarrier Communications: Prototype Filters, Lattice Structures, and Implementation Aspects," IEEE Communications Surveys Tutorials, vol. 16, no. 3, pp. 1312-1338, March 2014.

[23] J. A. Prakash and G. R. Reddy, "Efficient Prototype Filter Design for Filter Bank Multicarrier (FBMC) System Based on Ambiguity Function Analysis of Hermite Polynomials," in 2013 International Mutli-Conference on Automation, Computing, Communication, Control and Compressed Sensing. Kottayam, India: IEEE, March 2013, pp. 580-585.

[24] M. G. Bellanger, "Specification and Design of a Prototype Filter for Filter Bank Based Multicarrier Transmission," in 2001 IEEE International Conference on Acoustics, Speech, and Signal Processing. Proceedings, vol. 4. Salt Lake City, USA: IEEE, May 2001, pp. 2417-2420 vol.4.

[25] P. Siohan and C. Roche, "Cosine-Modulated Filterbanks Based on Extended Gaussian Functions," IEEE Transactions on Signal Processing, vol. 48, no. 11, pp. 3052-3061, 2000.

[26] A. Vahlin and N. Holte, "Optimal Finite Duration Pulses for OFDM," IEEE Transactions on Communications, vol. 44, no. 1, pp. 10-14, 1996.

[27] I. C.Moore and M. Cada, "Prolate Spheroidal Wave Functions, Fourier Analysis, and Uncertainty V: the Discrete Case," Prolate Spheroidal Wave Functions, An Introduction to the Slepian Series and Its Properties, vol. 16, no. 3, pp. 208-230, March 2004.

[28] J. Nadal, C. A. Nour, and A. Baghdadi, "Design and Evaluation of a Novel Short Prototype Filter for FBMC/OQAM Modulation," IEEE Access, vol. 6, pp. 19610-19625, 2018.

[29] A. Aminjavaheri, A. Farhang, L. E. Doyle, and B. Farhang-Boroujeny, "Prototype Filter Design for FBMC in Massive MIMO Channels," in 2017 IEEE International Conference on Communications (ICC). Paris, France: IEEE, May 2017, pp. 1-6.

[30] R. T. Kobayashi and T. Abrao, "FBMC Prototype Filter Design via Convex Optimization," IEEE Transactions on Vehicular Technology, vol. 68, no. 1, pp. 393-404, Jan 2019.

[31] T. Q. Nguyen and R. D. Koilpillai, "The Theory and Design of Arbitrary-Length Cosine-Modulated Filter Banks and Wavelets, Satisfying Perfect Reconstruction,” IEEE Transactions on Signal Processing, vol. 44, no. 3, pp. 473-483, March 1996.

[32] J. G. Proakis and D. G. Manolakis, Digital Communications, 4th ed. Upper Saddle River, USA: McGraw-Hill, 2000.

[33] K. Cho and D. Yoon, "On the General BER Expression of One- and Two-Dimensional Amplitude Modulations," IEEE Transactions on Communications, vol. 50, no. 7, pp. 1074-1080, July 2002.

[34] W. T. A. Lopes, F. Madeiro, and M. S. Alencar, "Closed-Form Expression for the Bit Error Probability of Rectangular QAM Subject to Rayleigh Fading,” in 2007 IEEE 66th Vehicular Technology Conference, September 2007, pp. 915-919. 
[35] J. E. Marengo, D. L. Farnsworth, and L. Stefanic, "A Geometric Derivation of the Irwin-Hall Distribution,” International Journal of Mathematics and Mathematical Sciences, vol. 2017, pp. 296-300, 2017.

[36] D. M. Bradley and R. C. Gupta, "On the Distribution of the Sum of n Non-Identically Distributed Uniform Random Variables," Annals of the Institute of Statistical Mathematics, vol. 54, no. 3, pp. 689-700, 2002.

[37] T. Ihalainen, T. H. Stitz, and M. Renfors, "Efficient Per-Carrier Channel Equalizer for Filter Bank Based Multicarrier Systems," in 2005 IEEE International Symposium on Circuits and Systems. Kobe, Japan: IEEE, May 2005, pp. 31753178 Vol. 4.

[38] D. S. Waldhauser, L. G. Baltar, and J. A. Nossek, "MMSE Subcarrier Equalization for Filter Bank Based Multicarrier Systems," in 2008 IEEE 9th Workshop on Signal Processing Advances in Wireless Communications. Recife, Brazil: IEEE, July 2008, pp. 525-529.

[39] T. Ihalainen, A. Ikhlef, J. Louveaux, and M. Renfors, "Channel Equalization for Multi-Antenna FBMC/OQAM Receivers," IEEE Transactions on Vehicular Technology, vol. 60, no. 5, pp. 2070-2085, June 2011.

[40] P. Siohan, C. Siclet, and N. Lacaille, "Analysis and Design of OFDM/OQAM Systems Based on Filterbank Theory,” IEEE Transactions on Signal Processing, vol. 50, no. 5, pp. 1170-1183, 2002. 